\title{
Orthodontic Treatment of Multiple Supernumerary \& Impacted Teeth: Interdisciplinary Approach
}

\author{
Patni VJ' Kolge $\mathrm{NE}^{1 *}$, Patkar $\mathrm{ND}^{2}$, Jain $\mathrm{MN}^{3}$, Ezzy $\mathrm{HK}^{4}$ and Kolge $\mathrm{GN}^{5}$ \\ ${ }^{1}$ Department of Orthodontics \& Dentofacial Orthopaedics, MGM Dental College \& Hospital, India \\ ${ }^{2}$ Department of Oral \& Maxillofacial Surgery, MGM Dental College \& Hospital, India \\ ${ }^{3}$ Department of Oral \& Maxillofacial Pathology, MGM Dental College \& Hospital, India \\ ${ }^{4}$ Department of Prosthodontics \& Crown and Bridge, MGM Dental College \& Hospital, India \\ ${ }^{5}$ Department of Conservative Dentistry \& Endodontics, Sinhgad Dental College \& Hospital, India
}

*Corresponding author: Neeraj E Kolge, Department of Orthodontics \& Dentofacial Orthopaedics, MGM Dental College \& Hospital, India

\begin{abstract}
The following case illustrates a patient with impacted maxillary central incisor along with multiple impacted supernumerary teeth. The patient presented for orthodontic treatment with the chief complaint of unesthetic appearance of his upper anterior teeth. Diagnosis, treatment planning, and various methods of managing guided eruption of impacted teeth are discussed.
\end{abstract}

Keywords: Odontoma; Supplemental supernumerary; Impacted Interdisciplinary

\section{Introduction}

Management of case is challenging with the presence of multiple supernumerary and impacted teeth and increases the treatment duration as well. Deciphering a proper treatment sequence, anchorage planning and execution of sound biomechanics is a challenging task in such situations. In this case report, we present a case of multiple supernumerary unerupted teeth without any associated abnormalities in a seventeen-year-old. Diagnosis was done while the patient was advised for routine X-rays prior to start of orthodontic treatment. Based on the clinical and radiological investigation, a diagnosis of idiopathic hyperdontia was made. Interdisciplinary approach is advocated in such cases.

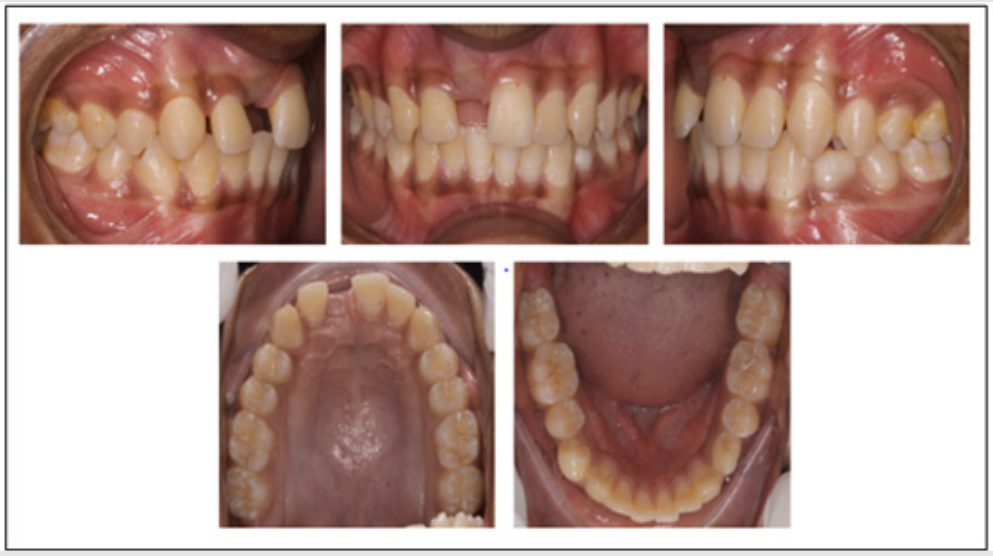

Figure 1: Pre-treatment Intra-oral Photographs. 


\section{Patient Information}

A 17-year-old male patient reported with a chief complaint of missing permanent right maxillary central incisor thus, compromised aesthetics. The patient presented with normal built, no previous medical and dental history and this was his first dental visit. The patient had no complaint of pain or signs of infection and was in good general health. The patient had a normal facial appearance and did not exhibit any physical or skeletal abnormality.

Intra-oral examination revealed a Class I molar and canine relationship bilaterally Figure 1, missing permanent right maxillary central incisor and a bulge which was felt on labial cortex in same region indicating presence of calcified mass. Over-retained deciduous lower left first molar. Minor rotational discrepancies were observed. Buccal /lingual mucosa, palate, tongue and floor of the mouth appeared normal.

\section{Diagnostic Assessment}

The patient was advised Orthopantomograph, Lateral Cephalograph; and IOPA radiographs with anterior teeth as a part of routine orthodontic radiographic examination. CBCT was carried out to evaluate the localization of the impacted teeth and calcified structures and to rule out any fusion of the supernumeraries to the permanent teeth in the maxillary and mandibular regions.

\section{Imaging}

a) OPG/ Lat Ceph: Radiographically, presence of irregular calcified mass (size: 1 x $1.5 \mathrm{~mm}$ ) in maxillary right posterior region Figure 2A, supernumerary tooth in maxillary anterior region Figure $2 \mathrm{~B}$ as well as bilateral tooth like structures in mandibular premolar region Figure 2C \& 2D were observed. CBCT scans of the maxilla and mandible were advised for localization of the impacted calcified structures. Cephalometrically, the maxilla and mandible were well positioned with respect to each other. A Class I apical base relationship was present (ANB: $+2^{0}$; SNA: $83^{0}$, SNB: $81^{\circ}$ ) with a horizontal growth pattern (FMA: $20^{\circ}$; Sum of angles: $381^{\circ}$ ) Figure 3. The patient was diagnosed Class I skeletal and dental malocclusion with mild arch length discrepancy and multiple impacted \& supernumerary teeth.

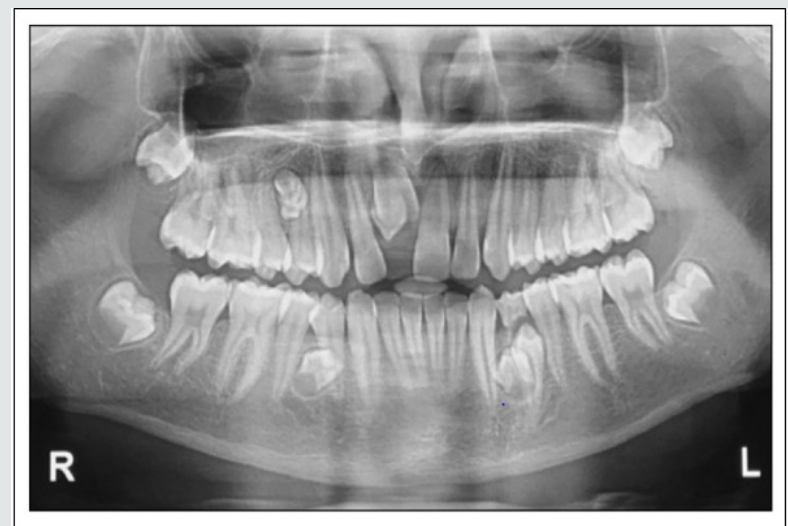

Figure 2: Presence of multiple supernumerary teeth on OPG

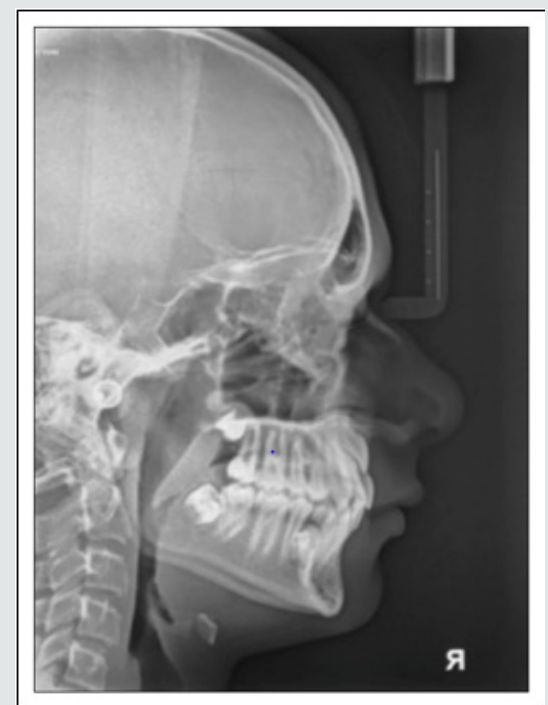

Figure 3: Pre-treatment Lateral Cephalo graph 


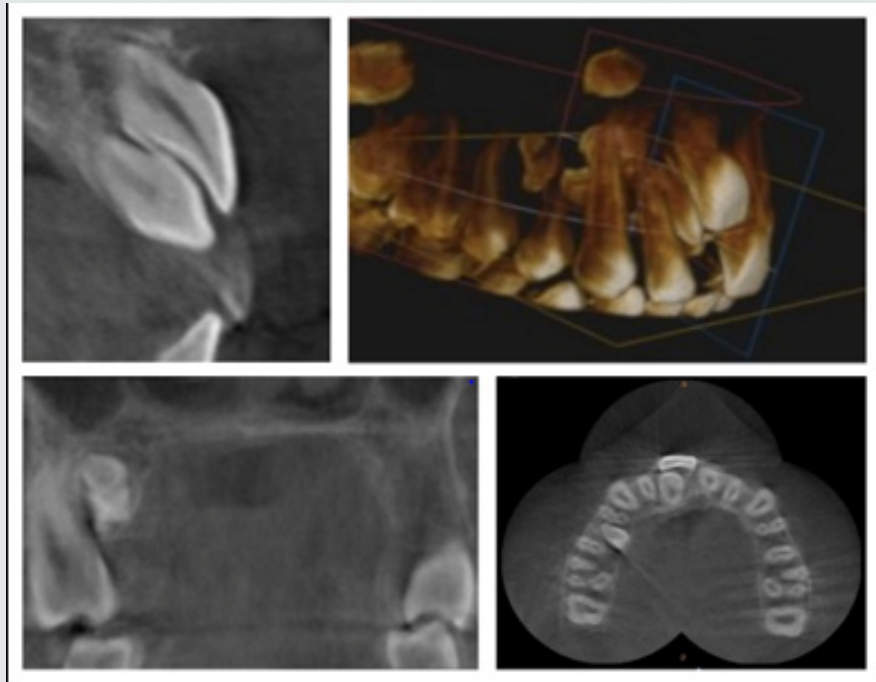

Figure 4: CBCT Scan (Maxilla).

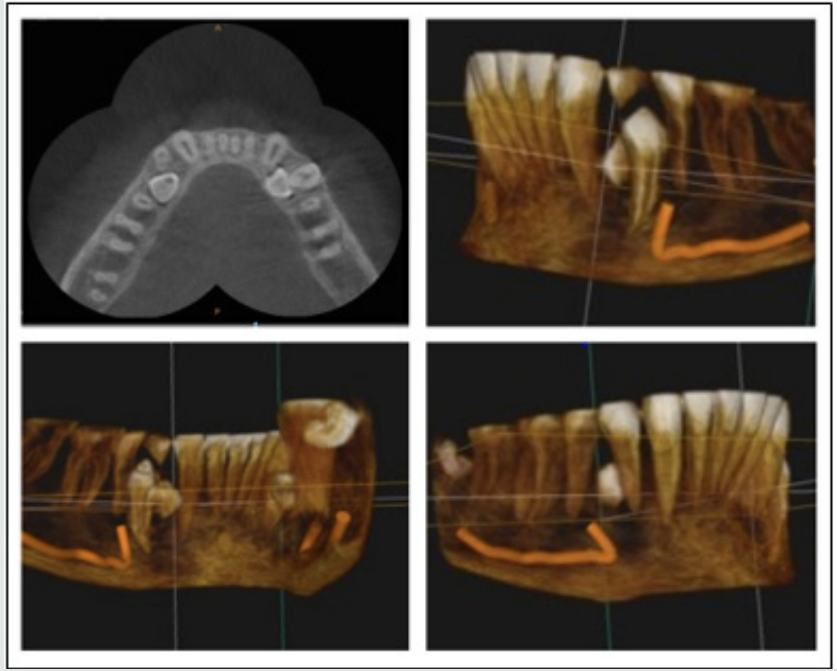

Figure 5: CBCT Scan (Maxilla).

b) CBCT: CBCT scans of maxilla and mandible revealed presence of irregular calcified masses w.r.t. upper right posterior quadrant. It also revealed impacted right permanent central incisor with a supernumerary tooth present palatal to the impacted tooth Figure 4. Impacted central incisor presented with a normal crown and under-developed root structure having a crown: Root ratio of approximately 1:1. There was minimal bone coverage with respect to the permanent central incisor. Presence of supplementary supernumerary teeth was also seen bilaterally in lower premolar region. They were located lingual to the permanent premolars; no fusion of any of the teeth or signs of disturbance to the path of eruption of the permanent teeth was observed Figure 5.

\section{Treatment Objectives}

The primary treatment objective was guided eruption of impacted maxillary central incisor. The orthodontic treatment plan necessitated fixed orthodontic therapy in coordination with oral surgery to expose and ligate the impacted teeth. Patient was informed about the fair prognosis of 11 . The patient expressed interest in orthodontic therapy for bringing the central incisor in place, but not surgical removal of other impacted teeth.

\section{Treatment Alternatives}

The patient had previously been informed that prosthodontic therapy might be his only option. A treatment alternative included extraction of the impacted permanent teeth and replacement with a fixed prosthesis. This remained a potential treatment alternative, however, as the patient was still young and concerned about cost and long-term replacement restorations that might be needed over his lifetime. The patient preferred the orthodontic approach as it had the potential to fully correct the problem. 


\section{Treatment Progress}

Prior to placing fixed orthodontic appliances, the patient underwent extraction of the calcified masses present in the maxillary tooth region and extraction with over-retained 74 Figure
6 \& 7. Orthodontic appliances were placed one week later. A 0.022 " $\mathrm{x} 0.028$ " slot MBT preadjusted edgewise appliance was bonded, and light continuous arch wires were placed. After four months with continuous arch wires, the patient was referred back to the oral surgeon with rigid 0.019 " x 0.025 " SS arch wires in place.

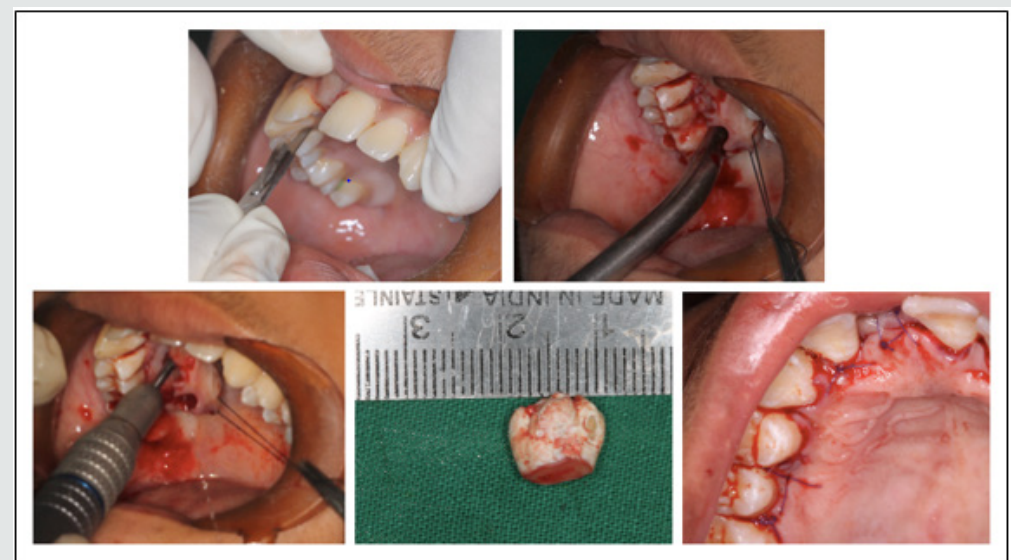

Figure 6: Extraction of supernumerary in right maxillary posterior region.

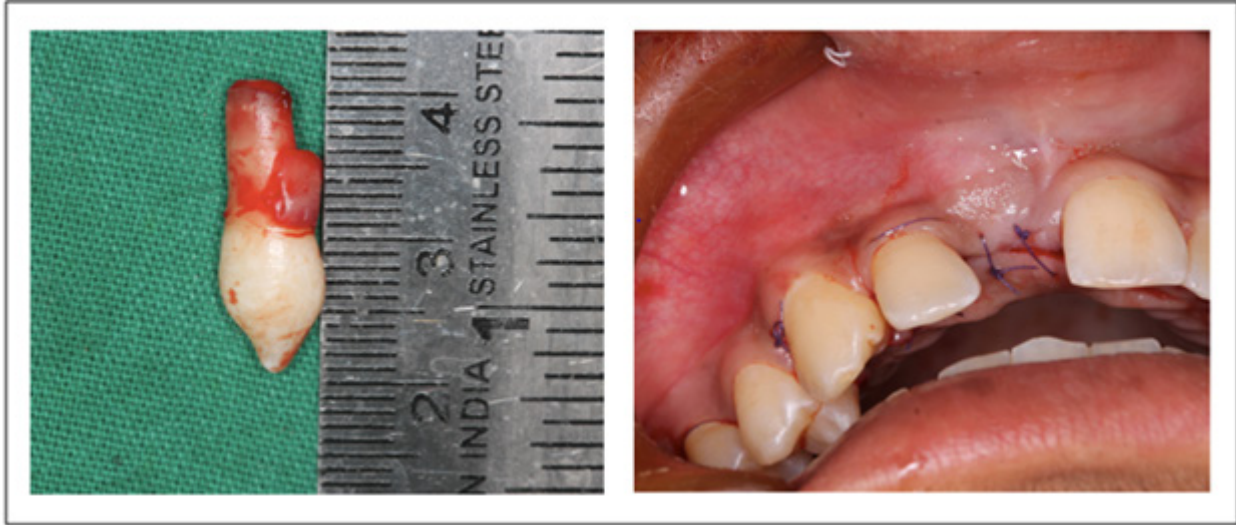

Figure 7: Extraction of supernumerary tooth palatal to impacted right central incisor.

Using crevicular incision, a full thickness mucoperiosteal flap was elevated. After achieving adequate exposure, supernumerary tooth palatal to the central incisor was luxated and extracted. The socket was thoroughly debrided using $5 \%$ betadine solution and normal saline. Wound closure was done using synthetic absorbable suture (3-0 vicryl). Using proper surgical principles, labial surface of maxillary central incisor was exposed; area was isolated, and a modified ribbon arch bracket bonded on the labial surface of the tooth. Power thread was placed from the bracket to the rigid arch wire incisal to the impacted tooth. The patient recalled at two and four weeks to replace the power thread.
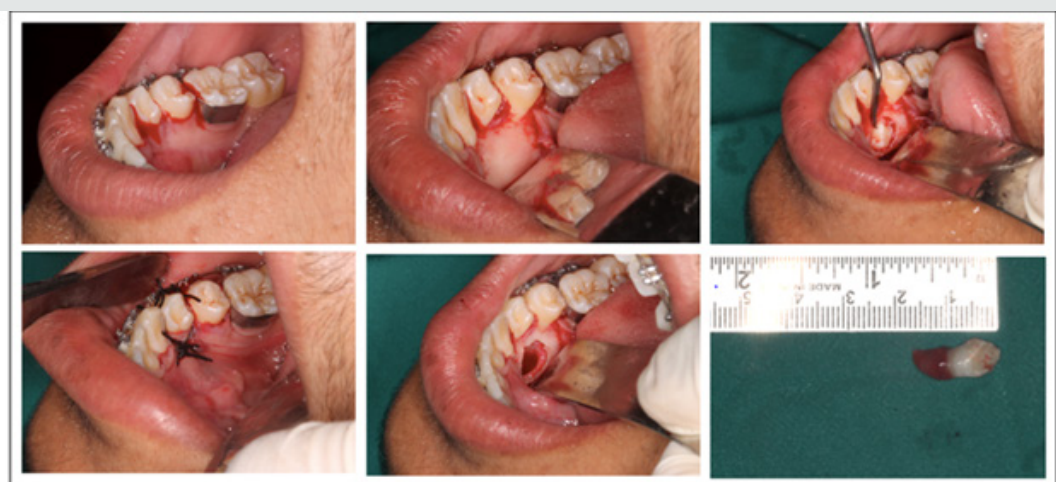

Figure 8: Supplementary premolar in the lower premolar region. 
One month after exposure and ligation, a 0.014" NiTi overlay wire was placed. After three months a continuous nickel titanium arch wire was placed in the maxillary arch. Torqueing auxiliary was placed on 11, for torque correction. Supplementary premolars in the lower premolar region were exposed lingually using full thickness mucoperiosteal flap. Adequate exposure was obtained using postage stamp technique and surgical extraction was carried out. Wound was thoroughly cleaned, debrided and sutured using synthetic absorbable suture (3-0 vicryl) Figure 8.

\section{Treatment Result}

At fifteen months, the patient was extremely pleased with the results that were obtained. Impacted Maxillary Central Incisor was levelled and aligned; a fixed lingual bonded retainer was planned to be placed bearing in mind the poor prognosis with the same. A well interdigitated Class I molar and canine relationship was obtained bilaterally.

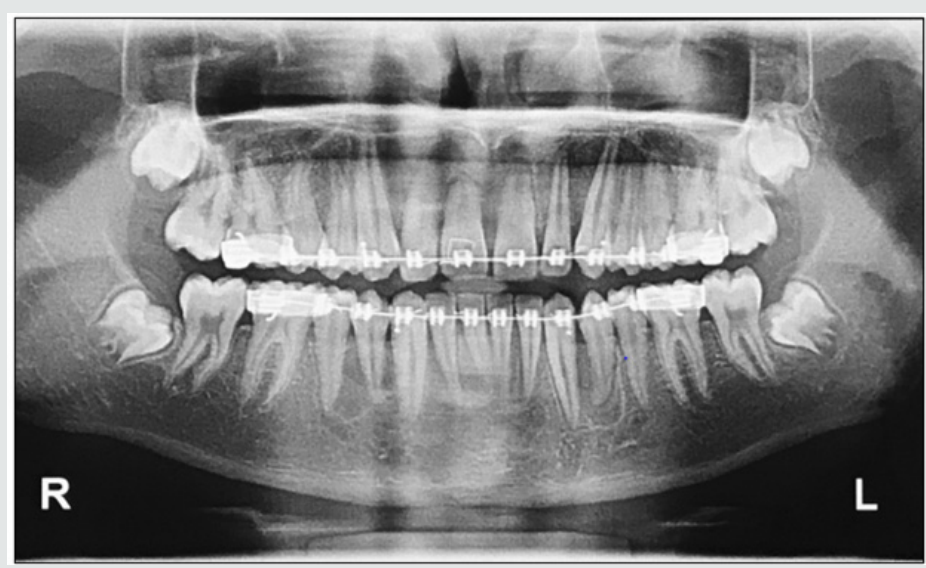

Figure 9: Post-surgical OPG

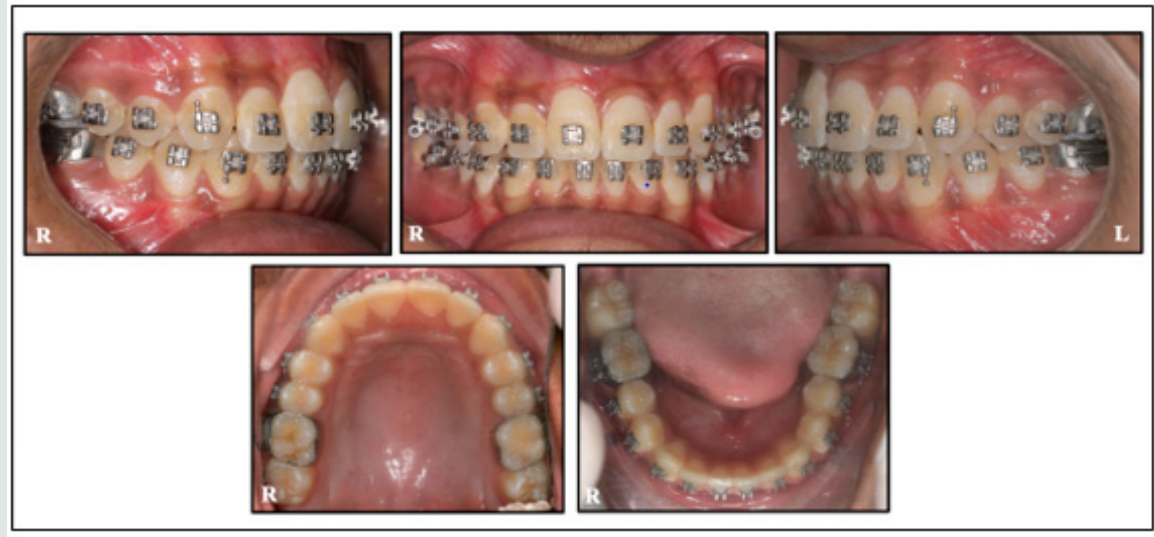

Figure 10: Pre-finishing Intra-oral Photographs.

A progress OPG Figure 9 was taken and the brackets repositioned accordingly. The final six months of treatment focused on achieving a well-inter-digitated posterior occlusion Figure 10.

\section{Discussion}

An otherwise straightforward/simple case can be made complicated by presence of impacted or supernumerary teeth. A thorough discussion with patient and the parents revealed their main concern being unsightly appearance in maxillary central incisor region due to impacted central incisor. Radiographic examination revealed presence of multiple impacted supernumerary teeth.

Supernumerary teeth are often detected during routine radiographic examination and can be found in any region of the jaw; erupted or non-erupted. Multiple supernumerary teeth can be associated with/without any underlying syndromes or diseases. Supernumerary teeth without associated syndromes is a rare phenomenon, as supernumerary teeth are usually associated with cleft lip/ palate and other syndromes such as cleidocranial dysplasia or Gardner's syndrome.

They are seen as a result of interference during the intial stages of development. Multivariate etiologies are observed; which can be phylogenetic relic of extinct ancestral tissue [1], dichotomy of the tooth bud [2] or hyperactivity of dental lamina [3]. Supernumerary teeth can be present in deciduous as well as permanent dentition and can manifest in way of crowding, impaction, delayed eruption or ectopic eruption. Supernumerary teeth can be classified according to their morphology, chronology, orientation and location [4]. classified them as single or multiple. Single supernumerary 
teeth are classified on the basis of their morphology as conical, tuberculate, supplementary, and odontomas; multiple being composite or complex [5]. classified supernumerary teeth as two types according to shape: supplementary or rudimentary; supplementary or eumorphic are those that have a normal shape and size and rudimentary or dysmorphic have an abnormal shape and smaller size and maybe conical, tuberculate, or molariform.

An impacted tooth is prevented from normal eruption due to obstruction in the eruptive pathway or lack of space in the arch. They may therefore be non-functional, abnormal or pathological. Local factors of impaction include ectopic position of tooth germ, crowding, supernumerary teeth, bony or soft tissue lesions. Many kinds of impactions include buccal, lingual, vertical, horizontal and inverted [6].

Various factors are to be taken into consideration for successful alignment of an impacted tooth. They are degree of root completion, presence of space for the impacted tooth in the arch and position and direction of the impacted tooth. Multiple methods [7] of applying eruptive force to the teeth can be utilized and are enumerated. A balance between each method's advantages and disadvantages should be sought out before adopting to a particular method.

Power thread provides very light eruptive forces, but force levels can decay quickly within a span of two weeks and they can no longer be sufficient to continue eruption thus making it time consuming for the patient to visit every two weeks. Nickel titanium overlay wires can be a good option for one impaction per arch/ quadrant. Inherent advantage of this system is presence of rigid arch wire as an anchor unit and flexible wire to deliver the eruptive forces. Continuous NiTi arch wires can be used as they are extremely flexible and can deliver an almost continuous force, but they do not provide necessary rigidity to the arch wire and thus undesirable side effects can be manifested. Vertical interarch elastics from an impacted tooth to the opposing arch work extremely well but depend on the patient compliance. Patients may forget to wear the elastics or may wear it in wrong direction. Also, auxiliary cantilevers can be useful in guided eruption but limitation being auxiliary tube to apply eruptive force along with a main arch wire tube to provide the necessary rigidity.

Frequently, on bringing the impacted central incisor into the arch, a discrepancy is seen in the gingival height in comparison to the adjacent incisor. Light forces are more effective than heavy forces in moving the impacted/ unerupted teeth and eventually a good gingival position and contour. In our case, the forces applied on the tooth were very light and in the range of 10-15 grams.

\section{Conclusion}

Supernumerary teeth can be present in any region of oral cavity. They can erupt or remain impacted and lead to complications. A clinician should formulate a sound treatment plan with prior discussion with patient and parents, to meet their expectations and other clinical challenges.

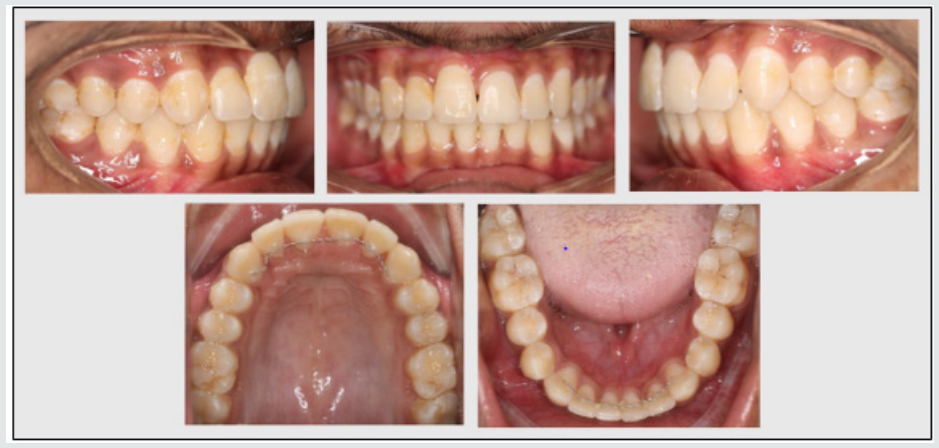

Figure 11: Post-treatment Intra-oral Photographs..

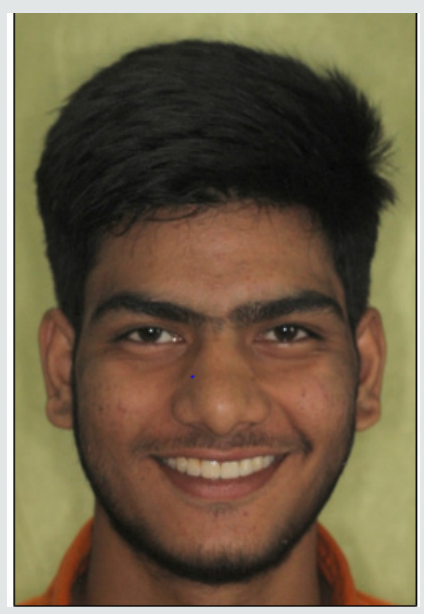

Figure 11: Post Treatment Extra-Oral Photograph.
Following orthodontic treatment that required extraction of multiple primary and permanent teeth as well as exposure and ligation of multiple permanent teeth, the treatment was completed with a significantly enhanced aesthetic result and improved selfconfidence Figure $11 \& 12$.

\section{Informed Consent}

The authors certify that they have acquired all necessary patient consent forms. Patient has given his consent for his images and other clinical information to be published in the journal, while their initials/ names will not be published and due efforts made to conceal their identity, though anonymity cannot be guaranteed.

\section{References}

1. Primo LG, Wilhem RS, Bastos EPS (1997) Frequency and characteristics of supernumerary teeth in Brazilian children: consequences and proposed treatments. Rev Fac Odont S Paulo 11(4): 231-237. 
2. Taylor GS (1972) Characteristics of supernumerary teeth in the primary and permanent dentition. Dent Pract Dent Record 22(5): 203-208.

3. Liu JF (1995) Characteristics of premaxillary supernumerary teeth: A survey of 112 cases. ASDC J Dent Child 62(4): 262-265.

4. Garvey MT, Barry HJ, Blake M (1999) Supernumerary teeth: An overview of classification, diagnosis and management. J Can Dent Assoc 65(11): 612-616.
5. Primosch RE (1981) Anterior supernumerary teeth: Assessment and surgical intervention in children. Pediatr Dent 3(2): 204-215.

6. Mwaniki D, Guthua SW (1996) Incidence of impacted mandibular third molars among dental patients in Nairobi, Kenya. Trop Dent J 19: 17-19.

7. Conley RS, Boyd SB, Legan HL, Jernigan CC, Starling C, et al. (2007) Treatment of a Patient with Multiple Impacted Teeth. Angle Orthod 77(4): 735-741.

\section{(c) (i) \\ This work is licensed under Creative Commons Attribution 4.0 License}

To Submit Your Article Click Here: Submit Article

DOI: $10.32474 /$ MADOHC.2019.03.000179

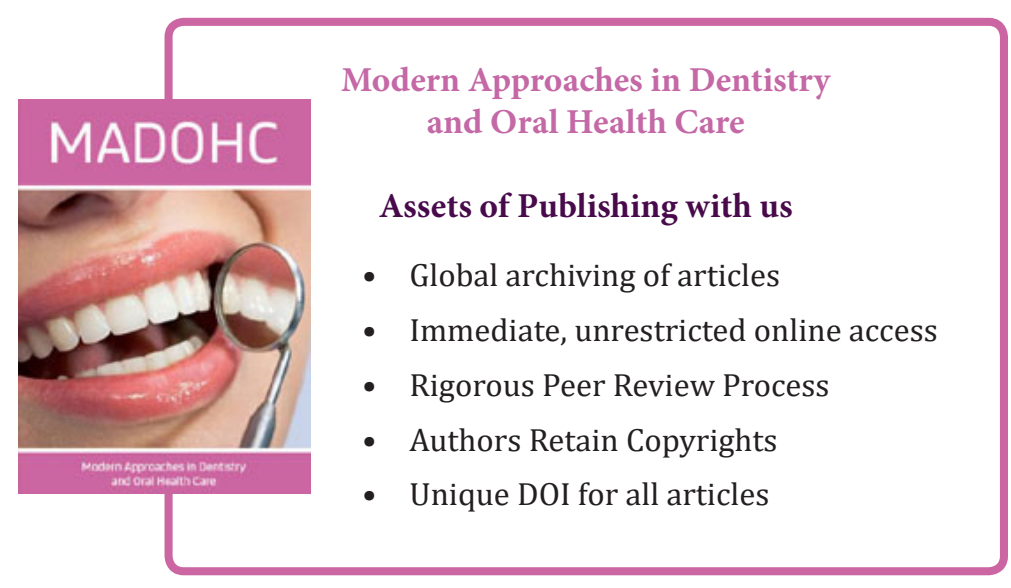

